\title{
The Effect of Transitional Care Mode on Exclusive Breastfeeding Rate of Gestational Diabetes Mellitus Patients
}

\author{
Zhili Chen ${ }^{1}$, Lin Wang ${ }^{1,}$, , Shiyi Zhang ${ }^{1}$, Yihong $W^{2}$ \\ ${ }^{1}$ Transitional Care Department, the First Affiliated Hospital of Jinan University, Guangzhou, China \\ ${ }^{2}$ Nursing College of Jinan University, Guangzhou, China
}

Email address:

chenzl1215@sina.com (Zhili Chen),wanglin18903078509@126.com (Lin Wang)

${ }^{*}$ Corresponding author

To cite this article:

Zhili Chen, Lin Wang, Shiyi Zhang, Yihong Wu. The Effect of Transitional Care Mode on Exclusive Breastfeeding Rate of Gestational Diabetes Mellitus Patients. American Journal of Nursing Science. Vol. 10, No. 3, 2021, pp. 151-155. doi: 10.11648/j.ajns.20211003.12

Received: March 23, 2021; Accepted: April 13, 2021; Published: April 29, 2021

\begin{abstract}
Objective: To explore the effect of transitional care mode on the exclusive breastfeeding rate of patients with gestational diabetes mellitus patients (GDM). Methods: A retrospective survey of patients who had undergone regular obstetrics and delivery in the hospital was carried out from December 2016 to December 2017. A random selection of 110 patients with GDM was included as the control group, and routine measures were taken. A total of 110 patients with GDM were selected as the experiment group. The whole nursing intervention was conducted with nurses in transitional care department on the basis of routine measures. The patients' knowledge about breastfeeding and the breastfeeding rate of the two groups were compared and analyzed. Results: A total of 220 patients with GDM were recruited. The awareness rate of breastfeeding could help to regulate glucose and lipid metabolism of mothers and newborns were higher than that in the control group $(P<0.001)$. For the issues related to breastfeeding, the experiment group was lower than the control group $(P<0.001)$. And after transitional care, the rate of exclusive breastfeeding was higher in the experiment group $(P<0.001)$. Conclusion: The postpartum transitional care mode can effectively increase the rate of postpartum exclusive breastfeeding of GDM patients and is worthy of reference by peers.
\end{abstract}

Keywords: Transitional Care Intervention, Gestational Diabetes, Exclusive Breastfeeding

\section{Introduction}

Gestational diabetes mellitus (GDM) is identified as the occurrence of first diagnosis of glucose tolerance abnormalities during pregnancy, not including the existed diabetes mellitus (DM)[1-2]. The GDM in one of the common complications during pregnancy. Zhu reported that among the 15,194 pregnant women who gave birth in 15 hospitals in China in 2012, the incidence of GDM accounted for $19.7 \%[3]$. With the policy opened to have a second child on January 2016 in China, more and more aging women planned to have two kids [4]. Thus, the proportion of GDM patients has increased significantly. A few studies showed that the breastfeeding can affect the postpartum glucose and lipid metabolism of GDM patients to lower blood glucose, reducing the risk of DM by $39.0 \% \sim 56 \%[5-6]$ in the future and pre-diabetes $44 \% \sim 86 \%[7-8]$. In 2019, the China Development Research Foundation released the Investigation Report on the Influencing Factors of Breastfeeding in China. According to the survey results, the exclusive breastfeeding rate of infants within 6 months was $29.2 \%$ [9]. Studies at home and abroad have found that the breastfeeding rate of GDM patients from 0 to 6 months postpartum shows a sharp downward trend with the passage of postpartum time [10-12]. Transitional care refers to a series of nursing behaviors by medical staff to ensure that patients receive different levels of coordinated and continuous health care in different places (such as from hospital to residence) and the same health care places (such as different departments of the hospital)[13]. The postpartum breastfeeding guidance for GDM patients is based on the conventional model, which focuses on health education during the hospitalization and before discharge. However, the education of breastfeeding for GDM patients is not enough to rely on the medical staff during hospitalization. The GDM patients will face a variety of 
problems after discharging from the hospital, which affects their confidence in breastfeeding. The transitional care model can enable GDM patients receive professional and continuity care during outpatient, hospitalization and residence to improve the self-efficacy of breastfeeding. In addition, it can also improve the rate of exclusive breastfeeding in patients with GDM patients after delivery. The author carried out transitional nursing intervention for patients with GDM to improve the rate of postpartum breastfeeding. The report is as follows.

\section{Data and Methods}

\subsection{General Information}

A retrospective survey was implemented from December 2016 to June 2017 to select 110 patients with GDM who delivered in the hospital and were given routine measures as the control group. 110 cases of GDM patients between July and December 2017 and were given transitional care were the experiment group. The patients with severe heart disease, hepatitis, kidney disease, mental illness and neonates suffering from phenylketonuria and lactose intolerance were excluded.

\subsection{Methods}

\subsubsection{The Routine Nursing Care Model}

The control group was implemented the routine nursing care during the hospitalization: (1) during the waiting delivery period: the breastfeeding health education was conducted by the nurse. (2) After delivery: the newborn would be given early contact, early feeding, early sucking and delayed cord clamping immediately after delivery. (3) During the hospitalization: the nurse in charge provided face to face breastfeeding guidance to the maternities. (4) Before discharge, the nurse would provide health education including breastfeeding, dietary guidance and neonatal care skills.

\subsubsection{The Transitional Care Model: The Experiment Group Implemented the Transitional Care Including Three Stage on the Basis of the Routine Care}

i. The case management during outpatient period

The patient diagnosed GDM in the outpatient were referred to the case manager by nurse for treatment.

ii. The case management during hospitalization period

Establishing postpartum health records of GDM patients. The postpartum health records of GDM patients were established by the full-time nurse of the International Board Certified Lactation Consultant (IBCLC) of the department of transitional care. The contents included name, diagnosis, height, body mass index (BMI), method of delivery, blood glucose, medication, breastfeeding assessment, etc., and developed a follow-up plan.

Strengthening the self-care ability of GDM patients. During the hospitalization, the IBCLC strengthened the "in-hospital" breastfeeding guidance on the basis of routine care; (a). Bedside health education about the breastfeeding method according to the patients' knowledge; (b). Give lectures about the knowledge of long-term and short-term benefits of breastfeeding, blood sugar control, medication, common breast problems during lactation that the patients and their families must participate together; (c). Provide the health education leaflets of postpartum knowledge for patients in order to consolidate theoretical knowledge. (d). The GDM patients generally had a heavy psychological burden, including their own diseases and the health of newborns. We can listen to the patients' complaints, assess their psychological problems and implement targeted psychological counseling and support. We inform the patients that breastfeeding will not transmit diabetes to the newborns, it is also one of the effective measures to prevent the development of type 2 diabetes. Encourage and guide family members to participate in breast-feeding, care more about patients and assist them in life care, and ensure that patients have enough sleep.

iii. The case management after discharge:

The breastfeeding consultation hotline was set up. The IBCLC of the transitional care department was responsible for answering the consultation calls after discharge, and providing professional guidance for the questions raised by the patients and their families.

We used the hospital follow-up system to establish a network of interactive communication for GDM patients. The IBCLC sent message 3 days, 1 week, and 1 month to the patients after discharge to provide lifestyle guidance such as diet, nutrition, exercise and breastfeeding, respectively.

The IBCLC provided home visits at the request of patients based on the results of telephone consultation. The nurse could provide individualized, face-to-face guidance for the patients and family members. The time of home visits were determined according to the patients' condition or willingness, and the numbers of visits should be increased based on the patients' and family members' mastery of breastfeeding knowledge and skills.

The nurse would make a telephone call after home visits. Generally, the first call was made on the second day after the visits, and the second call was made on the $10^{\text {th }}$ day. The purpose of the telephone call was to track the patients' and family members' knowledge and skills about breastfeeding and to correct the inappropriate ways. The nurse conducted telephone follow-up of 2 days, 2 weeks, 1 month, 3 months and 6 months after discharge and gave personalized professional guidance to strengthen breastfeeding confidence, and answer existing questions.

iv. Return to the hospital for visits

When returning to the obstetrics and gynecology education clinic after delivery for 42 days, the nurse in charge would give individualized breastfeeding guidance based on the status of the patients and the babies. If the nurse encountered different problems, the patients could be referred to the clinic of IBCLC in charge.

v. Re-examination of abnormal OGTT

If the patients diagnosed with abnormal OGTT after delivery for 6-12 weeks, they would be referred to the endocrinology department, and the IBCLC and the diabetes 
specialist nurse would follow-up their blood glucose and other conditions together.

\subsection{The Evaluation Index}

The investigator was responsible for telephone follow-up and inquiry to fill in the self-designed questionnaire after delivery for 1 month, 3 months, and 6 months. The questionnaire was designed by the researcher based on the relevant literature and content in the "Breastfeeding Training book". The questionnaire included the patient's general information, breastfeeding methods, knowledge, difficulties related to breastfeeding and skills at home.

\subsection{Data Analysis}

All data were analyzed using SPSS version 20.0 software. The quantitative data were expressed as the means \pm SD. The comparisons of quantitative data were conducted using the independent samples t-test, and categorical variables were compared using the chi-square test. $P<0.05$ was considered statistically significant.

\section{Results}

\subsection{Sample Description}

A total of 220 questionnaires were collected in the study and there were 220 valid questionnaires, Questionnaire-reclaiming efficiency was $100 \%$. Among them, 110 cases were conducted using the transitional care intervention, and 110 cases were conducted using routine nursing intervention.

\subsection{The Demographic and Clinical Features of the Patients and Newborns}

The demographic and clinical features of the patients and newborns were shown in Table 1.

Table 1. Characteristics of the female patients and newborns.

\begin{tabular}{lllllll}
\hline & n & Age (years) & Cesarean section & The weight of infants & Male & Term infants \\
\hline control group & 110 & $32.41 \pm 4.76$ & 44 & $3.22 \pm 0.43$ & 101 \\
experiment group & 110 & $33.36 \pm 4.54$ & 43 & $3.22 \pm 0.46$ & 49 \\
$T / \chi^{2}$ & 1.52 & 1.58 & 0.019 & 0.046 & 104 \\
$P$ & 0.466 & 0.083 & 0.890 & 0.545 & 0.605 \\
\hline
\end{tabular}

\subsection{The Comparison of the Two Groups of GDM Before and After Receiving the Transitional Care}

To compare the two groups of GDM patients before and after receiving the transitional care, the awareness rate of breastfeeding could help to regulate glucose and lipid metabolism of mothers and newborns were $93.63 \%$ and $92.73 \%$ in the experiment group respectively, which were much higher than that in the control group $(32.72 \%$ and $24.54 \%)$. The difference was statistically significant $(P<0.001)$. For the difficulties related to breastfeeding, the experiment group was lower than the control group (40.91\% vs $66.37 \%)$ and the difference was significant $(P<0.001)$ (Table 2). Besides, the breastfeeding alone of the experiment group was higher than that in the control group $(P<0.001)$ (Table 3).

Table 2. Comparison of breastfeeding-related knowledge between the two groups.

\begin{tabular}{llll}
\hline & $\mathbf{n}$ & $\begin{array}{l}\text { To regulate glucose and lipid } \\
\text { metabolism of mothers }\end{array}$ & $\begin{array}{l}\text { To regulate glucose and lipid } \\
\text { metabolism of newborns }\end{array}$ \\
\hline control group & 110 & $36(32.72 \%)$ & $27(24.54 \%)$ \\
experiment group & 110 & $103(93.63 \%)$ & $102(92.73 \%)$ \\
$\chi^{2}$ & & 85.1 & 102.12 \\
$P$ & & $<0.001 *$ & $<0.001 *$ \\
\hline
\end{tabular}

$* P<0.05$

Table 3. Comparison of breastfeeding alone between the two groups.

\begin{tabular}{lllll}
\hline & n & 1 month & 3 months & 6 months \\
\hline control group & 110 & $73(66.36 \%)$ & $67(60.91 \%)$ & $54(49.09 \%)$ \\
experiment group & 110 & $93(83.67 \%)$ & $89(80.70 \%)$ & $70(63.64 \%)$ \\
$\chi^{2}$ & & 9.82 & 10.67 & 4.73 \\
$P$ & & $0.002 *$ & $0.001 *$ & $0.030 *$ \\
\hline
\end{tabular}

$* P<0.05$

\section{Discussion}

\subsection{Importance and Necessity of Exclusive Postpartum Breastfeeding in Patients with GDM}

Breast milk is the best natural nutritional food for babies.
Breast milk also has great benefits for babies whose mothers diagnosed with GDM. The large amount of long-chain unsaturated fatty acids contained in breast milk can not only reduce insulin resistance, but also help maintain the blood glucose stability of newborns in the early postpartum period [14], and can reduce the risk of type 2 diabetes in the future. 
In addition, breastfeeding can reduce the probability of infant obesity, and the longer feeding time, the lower risk of obesity [15]. Therefore, breastfeeding has a great influence on the babies and GDM patients. However, the mothers not only has to experience physical changes, but also adapt to the challenge of taking care of the newborn. Thus, it is very easy to cause adverse influences of the body and psychology, such as poor rest, anxiety, and lack of confidence. Table 3 showed that the exclusive breastfeeding rate of the experimental group at 1,3 and 6 months postpartum was significantly higher than that of the control group $(\mathrm{P}<0.05)$. In this study, continuous nursing intervention was carried out for patients with GDM, which was a planned whole-process nursing management mode of "hospitalized - discharge". It focuses on maternal and child health, expert guidance, patient participation and dedicated management, which can significantly improve the postpartum breastfeeding rate of GDM patients.

\subsection{Transitional Care Is Conducive to Strengthening the Initiative of GDM Patients in Self-care}

Chertok [16] found that the breastfeeding self-efficacy scores of GDM patients were lower than those of normal patients. Some people with GDM worry that their high blood sugar, such as breast-feeding, will pass the "diabetes" to the baby and interfere with breast-feeding. For patients with GDM, childbirth involves meeting the challenges of physical changes and caring for the newborn. It is likely to cause adverse reactions in the patient's body, resulting in poor rest, anxiety, and lack of confidence in the breastfeeding child. So it will inhibit the pituitary gland secretion of prolactin and oxytocin.

Due to the lack of knowing the benefits of long-term and short-term benefits of breastfeeding, it is more likely to affect the effectiveness. During the hospitalization, some patients were unable to master the knowledge and skills of breastfeeding, and they were easily affected by the older generation felt insufficient milk secretion. As a result, the addition of formula milk gradually reduced the amount of breast milk, and finally had to replace breastfeeding with formula milk. In addition, disease factors and personal willingness also influenced the success rate of breastfeeding. Therefore, breastfeeding supportive intervention for GDM patients is essential. There were also existing other problems influencing the breastfeeding, including the nursing follow-up guidance, the nursing plan, lack of professional supportive in knowledge, continuous follow-up and the ability to deal with the problems.

In the study, the patients in the experiment group diagnosed in the outpatient would be referred to the case management of the ward. After delivery, the IBCLC assisted and provided professional guidance for patients to strengthen breastfeeding skills and knowledge and other aspects. After discharge, the hospital massage follow-up system, interactive platform communication, telephone follow-up and door-to-door visits were used to track the patients' situation. The postpartum transitional care intervention model guarantees the quality of care, fully motivates the patients and families in learning and helps to establish an effective family support network so that patients can receive help and support from family members in time when there is a problem. It provides support during the transition from passive care in the hospital to active care at home. In terms of transitional psychological care intervention, appropriated explanation and communication can reduce the tension and anxiety of patients and accelerate their adaptation to the role of mother. This may have a positive effect on reducing the incidence of postpartum depression. The results in Table 2 showed that the rate of breastfeeding related difficulties in the experimental group was significantly lower than that in the control group $(\mathrm{P}<0.001)$, and the awareness rate of breastfeeding to help maternal and infant regulate glucose and lipid metabolism was significantly higher than that in the control group $(\mathrm{P}<0.001)$. It is feasible and effective to provide whole process professional care for postpartum extended care and follow-up guidance outside the hospital, as well as targeted breastfeeding support, which can significantly improve the initiative of GDM patients and their families in self-care and reduce setbacks encountered during home breastfeeding.

\section{Conclusion}

In summary, in addition to conventional nursing measures, the postpartum transitional care intervention can find the accurate breastfeeding difficulties from gestational diabetes patients, improve the breastfeeding status of patients with GDM, reduce the risk of the future development of type 2 diabetes, and prevent the happening of the complications. On the basis of this study, an exclusive breastfeeding support system suitable for GDM patients is constructed, which can continue to improve the rate of exclusive breastfeeding in 6 months postpartum and the quality of life in postpartum。 It will benefit two generations.

\section{References}

[1] Yang Huixia, Xu Xianming, sun Weijie, et al. Guidelines for clinical practice of gestational diabetes mellitus (2nd Edition) [M]. People's Health Publishing House, 2013, 5 (2nd Edition): 64 .

[2] BASEVI V. Diagnosis and classification of diabetes mellitus [J]. Diabetes Care, 2011, 34 (S1): S62 - S69.

[3] Zhu WW, Yang HX, Wang C, et al. High prevalence of gestational diatebesmellitus in Beijing: effect of maternalbirth weight and other risk factors [J]. China Med J, 2017, 130 (9): 1019-1025.

[4] Zhai ZW, Li L, Chen JJ. Target population and new birth population estimation under the comprehensive two-child policy [J]. Population Research, 2016, 40 (4): 35-51.

[5] Slusser W M. Breastfeeding and maternal and infant health outcomes in developed countries [J]. Aap Grand Rounds, 2007, 18 (2): 15-16. 
[6] Oreilly M W, Abalos G, Dennedy M C, et al. Atlantic DIP: high prevalence of abnormal glucose tolerance post partum is reduced by breast-feeding in women with prior gestational diabetes mellitus [J]. European Journal of Endocrinology, 2011, 165 (6): 953-959.

[7] Ziegler A, Wallner M, Kaiser I, et al. Long-Term Protective Effect of Lactation on the Development of Type 2 Diabetes in Women With Recent Gestational Diabetes Mellitus [J]. Diabetes, 2012, 61 (12): 3167-3171.

[8] Trout K K, Averbuch T, Barowski M. Promoting Breastfeeding Among Obese Women and Women with Gestational Diabetes Mellitus [J]. Current Diabetes Reports, 2010, 11 (1): 7-12.

[9] China Development Research Foundation. The investigation report on influencing factors of breastfeeding in China [EB/OL], 2019-02-25.

[10] Yin Yanan, Luo Biru. Research progress of breast feeding [J]. Chinese Journal of maternal and child clinical medicine, 2013, 06: 837-841.

[11] Soltani H, DickinsonI F M, Kalk J, et al. Breast feeding practices and views among diabetic women: a retrospective cohort study [J]. Midwifery, 2008, 24 (4): 471-479.
[12] Zhang Shaomin. Breast feeding status and influencing factors of gestational diabetes mellitus patients at different stages of postpartum [J]. China maternal and child health care, 2015, 33: 5765-5767.

[13] Jin Jia, Huang Lihua. Progress in the research of the continuing nursing of gynecological cancer survivors [j]. Chinese Journal of nursing, 2017, 52 (5): 598-603.

[14] Chertok I R A, Raz I, Shoham I, et al. Effects of early breastfeeding on neonatal glucose levels of term infants born to women with gestational diabetes [J]. Journal of Human Nutrition and Dietetics, 2009, 22 (2): 166-169.

[15] Weyermann M, Rothenbacher D, Brenner H. Duration of breastfeeding and risk of overweight in childhood: a prospective birth cohort study from Germany [J]. International Journal of Obesity, 2006, 30 (8): 1281-1287.

[16] Chertok I R, Sherby E. Breastfeeding Self-efficacy of Women With and Without Gestational Diabetes [J]. MCN: The American Journal of Maternal/Child Nursing, 2016, 41 (3): 173-178. 